\title{
Ultrasonography of the pediatric hip and spine
}

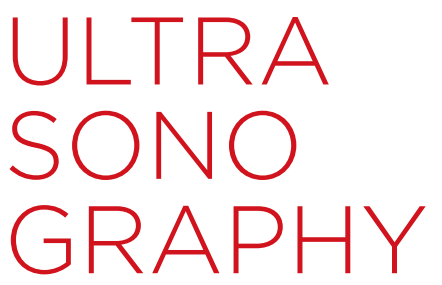

\author{
Yeo Ryang Kang, Joonbum Koo \\ Department of Radiology, Dongguk University Ilsan Hospital, Dongguk University College of \\ Medicine, Goyang, Korea
}

Ultrasonography (US) is a useful screening method for the diagnosis of developmental dysplasia of the hip (DDH) and congenital spinal anomalies in infants. In addition, US is a useful, noninvasive imaging modality for the diagnosis of transient synovitis in children. The early detection of DDH leads to a better prognosis without surgical intervention. Moreover, spinal US allows the detection of congenital malformations and can also demonstrate normal anatomy and normal variants that may resemble disorders. Therefore, radiologists should be familiar with the sonographic anatomy of the normal infant hip, scanning and measurement techniques for DDH, the US features of transient synovitis in children, spinal US techniques, and the US features of normal anatomical structures, normal variants, and congenital spinal anomalies.

Keywords: Ultrasonography; Hip dislocation, congenital; Spinal Dysraphism

\section{Introduction}

Developmental dysplasia of the hip (DDH) is one of the most common causes of disability among children. DDH encompasses a wide variety of pathologic conditions, ranging from fine acetabular dysplasia to irreducible hip dislocation. The previous term congenital dysplasia of the hip has been replaced by developmental dysplasia of the hip, because many of the clinical manifestations of DDH may not be detectable at birth, but are recognized at a later age [1].

The incidence of DDH ranges from 1.5 to 20 per 1,000 births. Multiple risk factors have been described, including breech positioning in utero, being the first-born child, oligohydramnios, family history, female sex, and deformities (postural or structural) of the foot and torticollis. In addition, increased joint laxity in the setting of exposure to maternal estrogens during the perinatal period may play a role in the development of $\mathrm{DDH}$, and the left hip is more frequently affected than the right $[2,3]$.

Ultrasonography (US) is the preferred modality for evaluating the hip in infants aged less than 6 months. US enables dynamic evaluation of the hip with stress maneuvering, as well as direct imaging of the cartilaginous portions of the hip that cannot be seen on plain radiographs [4]. Hip US has become the most commonly used diagnostic tool for DDH during early infancy because the early and accurate diagnosis of DDH is the most important factor contributing to appropriate treatment [5].

Additionally, US can thoroughly characterize nearly all spinal anomalies during the first days of life. To understand spinal anomalies, a knowledge of embryonic development is necessary. Starting on the 17th day of gestation, the neural plate thickens bilaterally to form the neural folds. During normal development, the neural folds close in toward the midline to form the neural tube. Premature

\section{REVIEW ARTICLE}

https://doi.org/10.14366/usg. 16051 pISSN: 2288-5919 • eISSN: 2288-5943 Ultrasonography 2017;36:239-251

Received: December 24, 2016 Revised: February 22, 2017 Accepted: February 22, 2017

Correspondence to: Joonbum Koo, MD, PhD, Department of Radiology, Dongguk University Ilsan Hospital, Dongguk University College of Medicine, 27 Dongguk-ro, Ilsandong-gu, Goyang 10326, Korea

Tel. +82-31-961-7826

Fax. +82-31-961-7851

E-mail: drkoo1@daum.net

\begin{abstract}
This is an Open Access article distributed under the terms of the Creative Commons Attribution NonCommercial License (http://creativecommons.org/ licenses/by-nc/3.0//) which permits unrestricted noncommercial use, distribution, and reproduction in any medium, provided the original work is properly cited.
\end{abstract}

Copyright (C) 2017 Korean Society of Ultrasound in Medicine (KSUM)

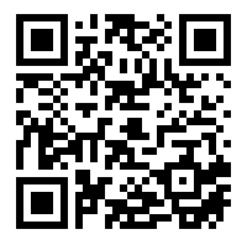

How to cite this article:

Kang YR, Koo JB. Ultrasonography of the pediatric hip and spine. Ultrasonography. 2017 Jul;36(3):239-251. 
disjunction of the neural ectoderm from the cutaneous ectoderm results in the development of spinal dysraphism, which is defined as incomplete or absent fusion of the midline neural, mesenchymal, and cutaneous structures and can be classified into three categories: spina bifida aperta, occult spinal dysraphism, and caudal spinal anomalies $[6,7]$.

As the ultrasound beam passes through predominantly cartilaginous and incompletely ossified spinal arches in newborns and infants, US can be used to investigate both the spinal canal and cord. Progressive ossification of the posterior elements of the vertebrae hinders satisfactory examinations in older children [6].

This article describes the anatomy of the normal infant hip joint, procedural details for performing US, landmarks, the classification of US results, the measuring technique, and the US appearance of transient synovitis in older children. Furthermore, we describe the technique for performing spinal US in newborns, the anatomy of the normal infant spine, normal variants, and congenital spinal anomalies.

\section{Developmental Dysplasia of the Hip}

\section{US Technique and Anatomy of the Normal Infant Hip Joint}

The Graf method is perhaps the most widely used US screening technique. If the well-established techniques for examination, interpretation, and measurement are meticulously followed, it is easy to manage newborn hip problems via this method [5].

To perform hip US, the hip joints must be evaluated in the standard coronal plane with a linear array probe [8]. Before evaluation of the hip joint, it is essential to identify the chondroosseous junction. This is because the echo of the chondro-osseous junction is an important landmark used to identify the femoral neck and other anatomical landmarks: the femoral head, iliac bone, lower limb of the ilium, acetabular bony roof, cartilaginous acetabular roof, acetabular labrum, joint capsule, and synovial fold (Fig. 1) [8].

\section{Scanning Technique}

The coronal view can be obtained with the hip in either the physiologic neutral position $\left(15^{\circ}-20^{\circ}\right.$ flexion) or the flexed position. The ultrasound transducer is then placed in the anatomic coronal plane (Fig. 2A). Next, the transducer is moved backwards and forwards from the basic position to identify the round structure of the hip joint. If the superior edge of the transducer is rotated posteriorly by $10^{\circ}$ to $15^{\circ}$ into an oblique coronal plane, the ilium will appear to be straight (Fig. 3A, C). If a sonogram contains a straight iliac wing contour, triradiate cartilage, and an apparent acetabular labrum, this indicates that it has a standard plane (Fig. 2B) $[8,9]$. However, in dislocated hips, lateral and posterior displacement of the femoral head prevents visualization of the femoral head and the center of the acetabulum in the standard plane. Therefore, if the displaced femoral head is followed, the ultrasound plane is no longer in the standard plane. The direction of displacement of the femoral head means that it is usually the posterior sectional plane that is visible (Fig. 3A, D) [8].

The American College of Radiology also recommends that a standard ultrasound examination be performed in the transverse view with the hip flexed at $90^{\circ}$ (Fig. 4A). The femoral shaft is seen anteriorly, terminating in the femoral head, which rests on the ischium (Fig. 4B). To identify a dislocatable hip, the transducer is placed in a posterolateral position during the Ortolani and Barlow maneuvers. If the relationship between the posterior acetabulum and the femoral head changes with gentle stress, the hip is unstable [9].

\section{Measurement Technique}

Three anatomic landmarks (the iliac line, triradiate cartilage, and labrum) are used to measure the $a$ and $\beta$ angles. Meticulous measurement of the $\alpha$ angle is necessary because false-positive findings can occur in the anterior or posterior sectional planes (Fig. $3 B, D)$. The a angle should not be determined as the average angle, but rather as the largest angle [2].

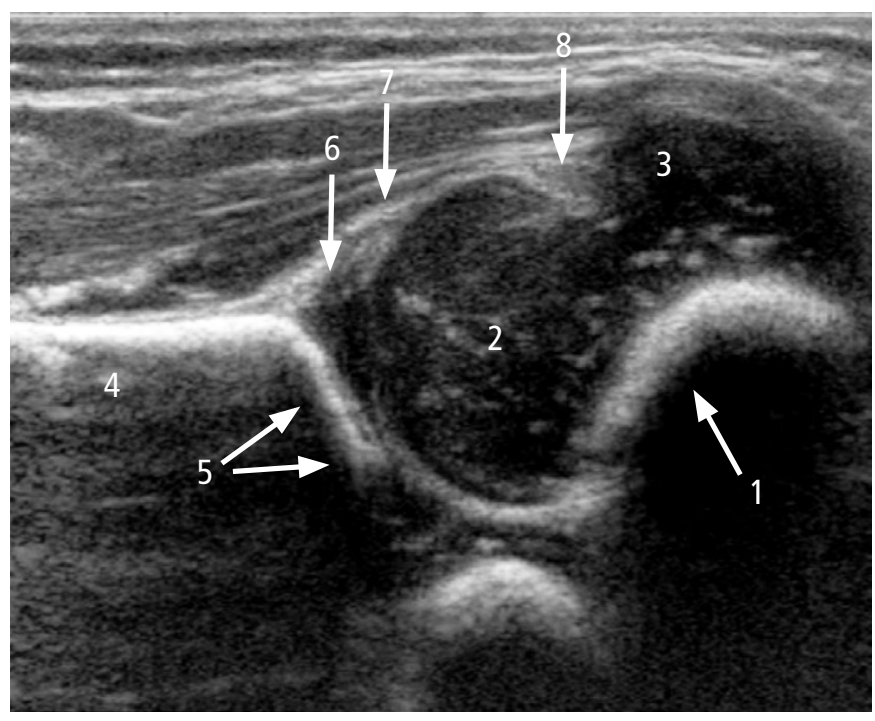

Fig. 1. A lateral coronal ultrasonography image through the right hip joint of a 4-week-old girl with a normal clinical hip examination. 1, chondro-osseous junction between the bony part and the cartilaginous part of the femoral neck; 2, cartilaginous part of the femoral head (hyaline cartilage); 3 , greater trochanter; 4 , iliac bone; 5 , lower limb of the ilium and bony acetabular roof; 6 , cartilaginous acetabular roof; 7 , acetabular labrum; 8 , synovial fold. 


\section{Graf a angle}

The Graf $a$ angle is defined as the angle formed between the acetabular roof and the vertical cortex of the ilium in the coronal plane (Fig. 5A). An a angle less than $60^{\circ}$ is abnormal and reflects a shallow acetabulum.

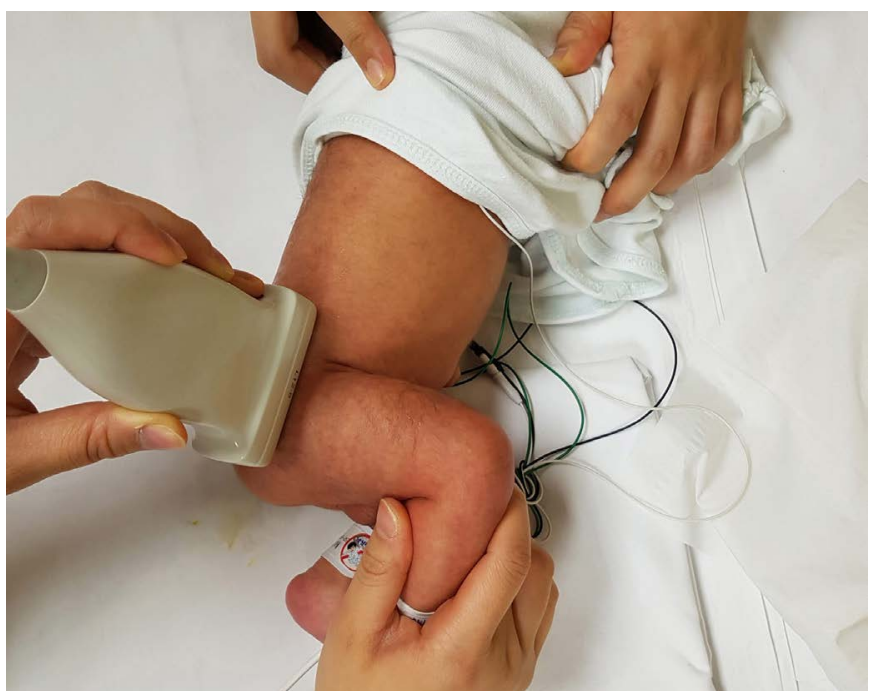

\section{A}

\section{Graf $\beta$ angle}

The Graf $\beta$ angle is formed by a line drawn through the vertical ilium and the cartilaginous acetabular labrum (Fig. 5A). A Graf $\beta$ angle greater than $55^{\circ}$ is abnormal. With superolateral femoral head displacement, the labrum is elevated, thereby increasing the $\beta$ angle $[2,8]$.

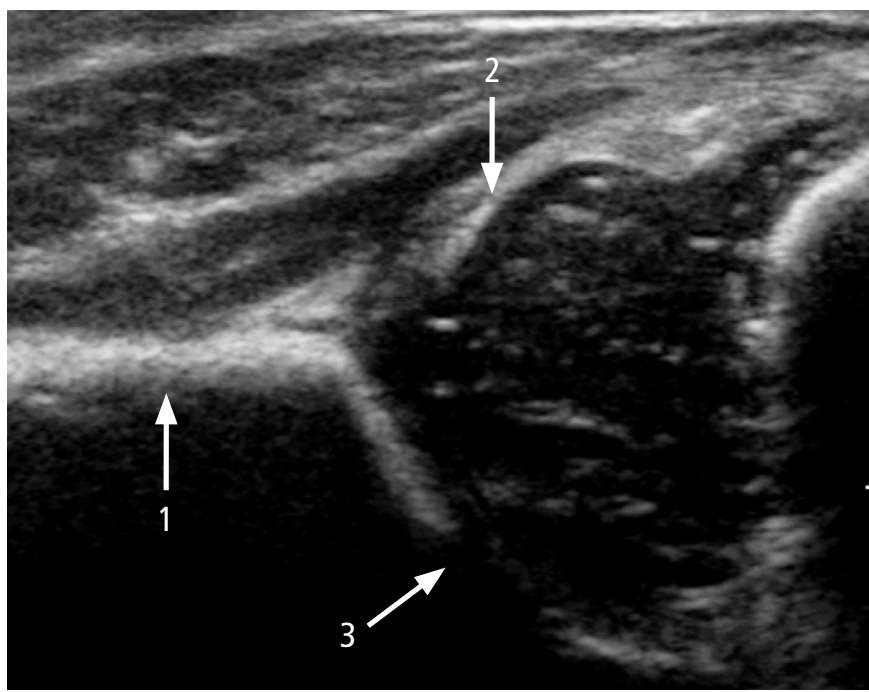

B

Fig. 2. A standard coronal ultrasonography through the right hip joint of a 5-week-old boy with a normal clinical hip examination. A. Ultrasound linear transducer is placed parallel to the lateral aspect of the infant's hip. B. Ultrasonography of the infant hip in the coronal plane has three landmarks: 1, a straight iliac line; 2, the tip of the acetabular labrum; and 3, the transition from the os ilium to the triradiate cartilage.

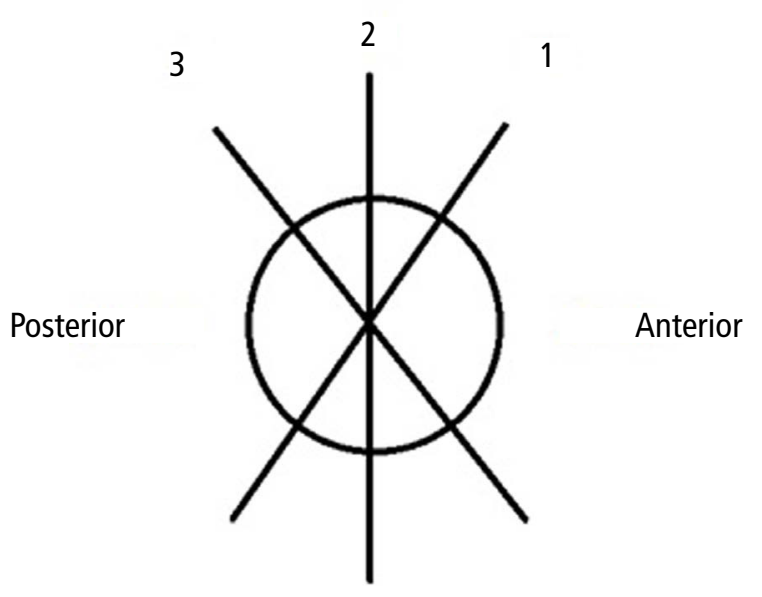

A

Fig. 3. Schematic image of the acetabular roof and US image through the left hip joint of a 6-month-old girl with a normal clinical hip examination.

A. Schematic image of the acetabular roof shows anterior sectional (1), standard (2), and posterior sectional planes (3). B. In the anterior sectional plane, the silhouette of the iliac bone bends to the anterior, i.e., towards the probe. 


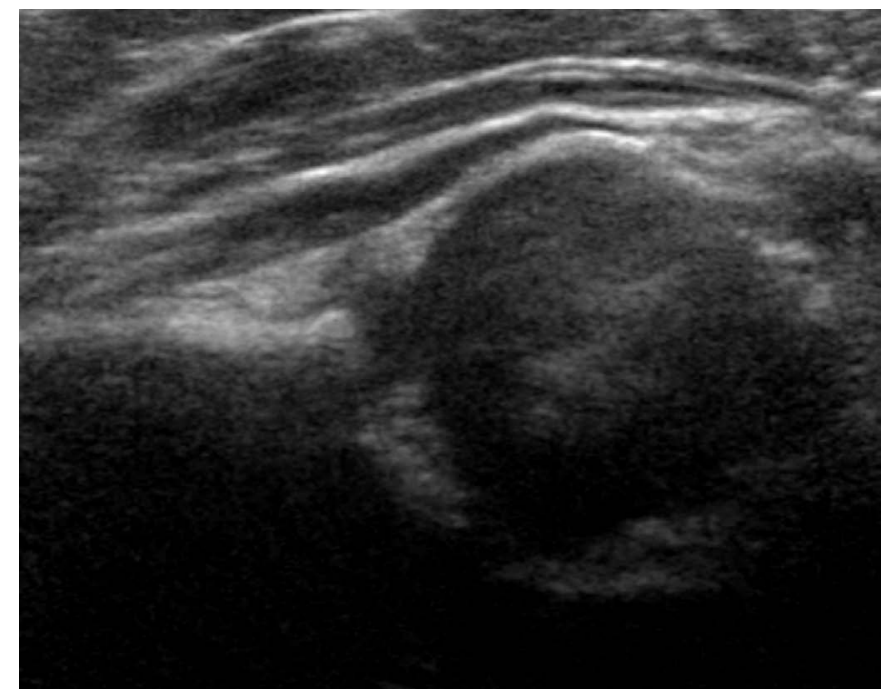

C

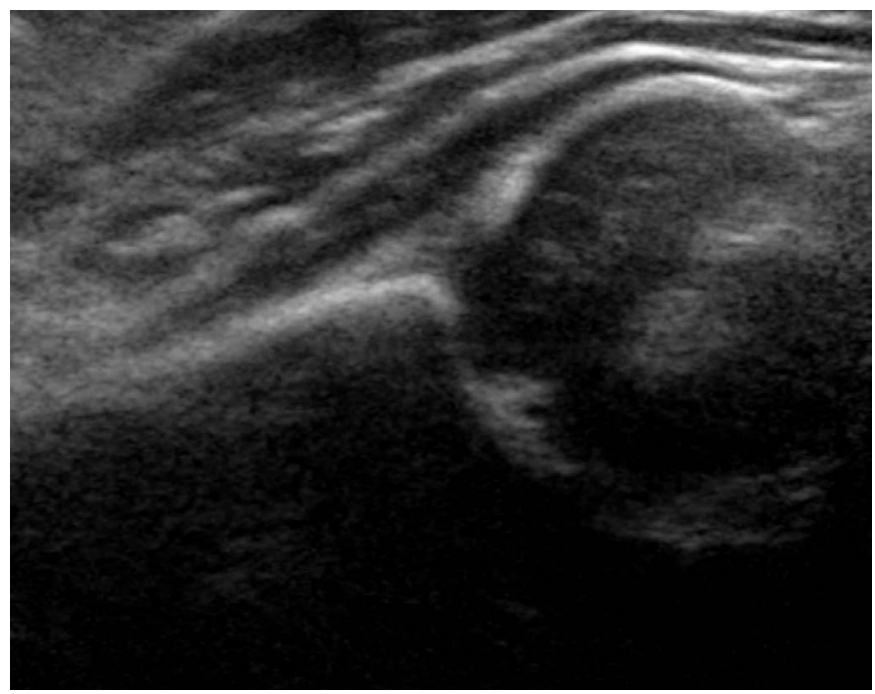

D

Fig. 3. C. In the middle sectional planes (the standard plane), the contour and the silhouette of the iliac bone are straight and parallel to the probe. D. In the posterior sectional plane, the silhouette of the iliac bone in the posterior sectional plane bends to the posterior, away from the probe.

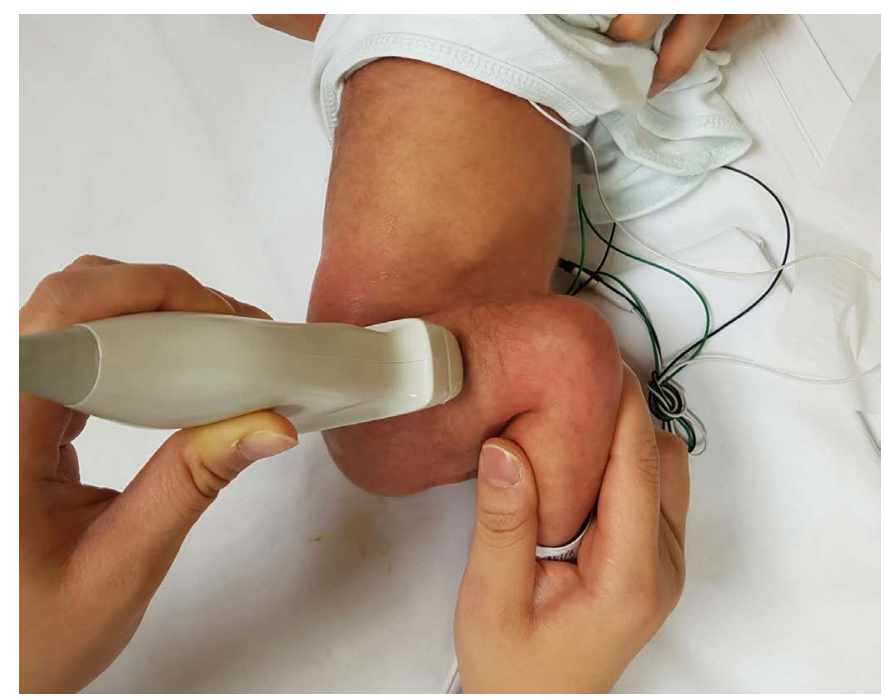

A

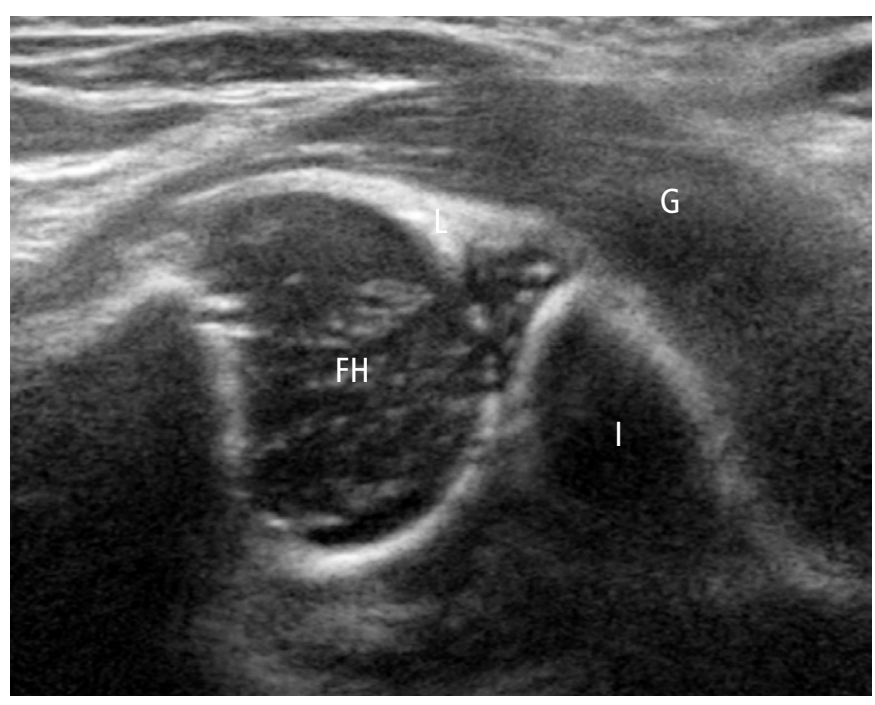

B

Fig. 4. A transverse flexion ultrasonography through the right hip joint of a 5-week-old boy with a normal clinical hip examination.

A. The hip and knee are flexed $90^{\circ}$, and the ultrasound transducer is placed perpendicular to the lateral aspect of the infant's hip. B. Transverse ultrasound image shows gluteus muscles (G), the cartilaginous femoral head (FH), metaphysis, ischium (I), and labrum (L).

\section{Femoral head position relative to the acetabulum}

A normally positioned femoral head is more than $50 \%$ covered by the acetabulum (Fig. 5B). DDH results in a shallow acetabulum and decreased coverage of the femoral head $[2,8]$.

\section{Graf sonographic hip classification}

The Graf classification method is based mainly on the a angle. Other parameters, such as the age of the patient, $\beta$ angle, course of the perichondrium of the cartilage acetabular roof, and structural changes in the cartilage roof, indicate specific variations (Table 1) [5].

An a angle greater than $60^{\circ}$ is graded as a normal hip (type I); these patients require no treatment [4]. If the $a$ angle is $50^{\circ}-$ $59^{\circ}$ in infants less than 3 months of age (Fig. 6), it is considered a type lla immature hip; that is, the hip is normally located, but the bony acetabulum promontory is rounded. These patients require no treatment, although there is a small risk of delayed DDH. Follow- 


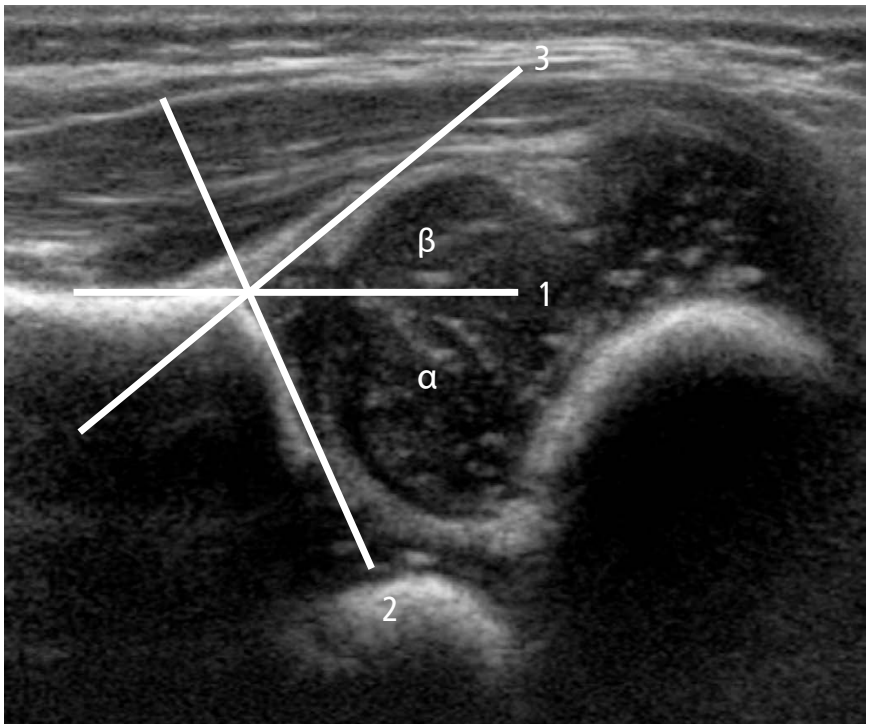

A

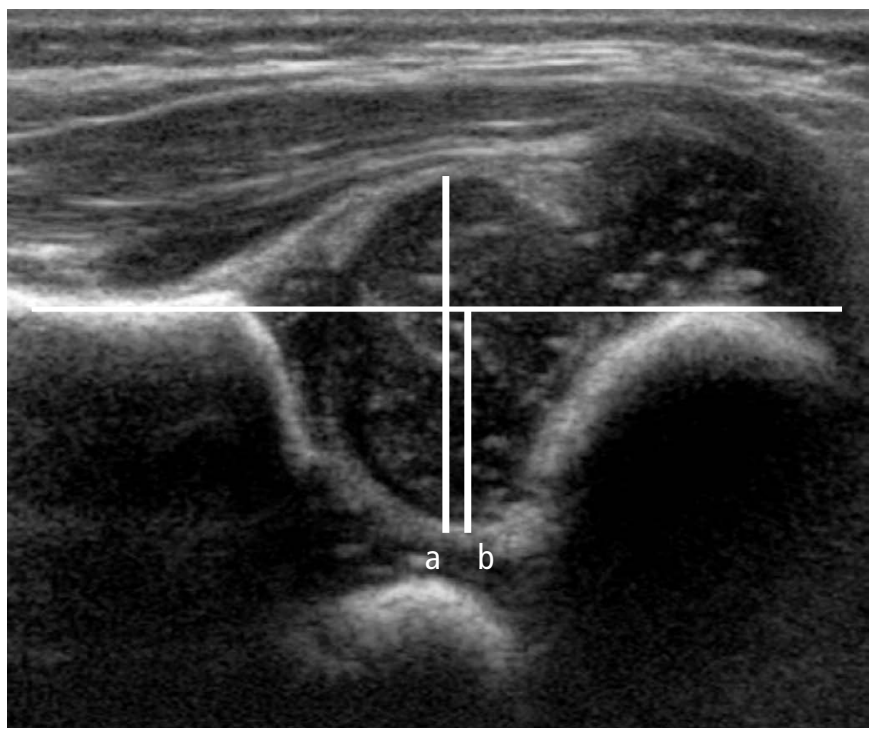

B

Fig. 5. Lateral coronal ultrasonography through the right hip joint of a 4-week-old girl with a normal clinical hip examination.

A. First, the baseline (1) runs along the lateral straight portion of the ilium. Second, the bony roof line (2) is drawn tangentially from the lower limb of the os ilium to the bony rim. Finally, the cartilage roof line (3) is drawn from the bony rim through the center of the acetabular labrum. B. Lateral coronal view through the right hip joint shows measurement of femoral head coverage. b/a $\times 100$, the percentage of the femoral head lying medial to the lateral iliac border.

Table 1. Sonographic anatomic classifications according to the Graf method

\begin{tabular}{|c|c|c|c|}
\hline Type & a angle $\left({ }^{\circ}\right)$ & $\beta$ angle $\left({ }^{\circ}\right)$ & Description \\
\hline I & $\geq 60$ & $<55$ & $\begin{array}{l}\text { Normal, fully mature hip. The acetabular rim is angular and the acetabular cup is deep. The cartilaginous roof covers the } \\
\text { femoral head. }\end{array}$ \\
\hline Ila & $50-59$ & $55-77$ & Physiologically immature at less than 3 months of age. \\
\hline$\| \mathrm{b}$ & $50-59$ & $55-77$ & $\begin{array}{l}\text { Similar to lla but in infants older than } 3 \text { months. The joint is dysplastic and requires treatment to prevent further } \\
\text { deterioration and dislocation. }\end{array}$ \\
\hline$\| c$ & $43-49$ & $<77$ & The hip socket is severely dysplastic, and is close to decentering, but the cartilaginous roof still covers the femoral head. \\
\hline$\| d$ & $43-49$ & $>77$ & Similar to llc but the hip is decentered. The cartilaginous roof bends cranially. \\
\hline III & $<43$ & $>77$ & Dislocated femoral head with a shallow acetabulum. \\
\hline IV & $<43$ & $>77$ & Dislocated femoral head with a severely shallow, dysplastic acetabulum. The cartilaginous roof is markedly displaced. \\
\hline
\end{tabular}

up at the age of 3 months is recommended to confirm normal development [4]. A type IIb hip has similar features to type lla but is detected in children more than 3 months of age with variable degrees of subluxation in the neutral position and/or during the Barlow maneuver. Types IIc, IId, III, and IV represent progressively abnormal hips with frank subluxation occurring in types III and IV. The a angle is $<50^{\circ}$ in types IIC (Fig. 7) and IId and $<43^{\circ}$ in types III and IV [5].

\section{Treatment of DDH}

The current gold-standard treatment for DDH in newborns to infants aged 6 months remains the Pavlik harness. The Pavlik harness provides the best results, with a success rate of $85 \%-95 \%$, and thus should be started as early as possible to achieve the best outcome. The purpose of the harness is to maintain the hip in a flexed and abducted position in order to bring the femoral head as close to the acetabular ring as possible [1].

Between 6 months and 2 years of age, closed reduction and casting is attempted under general anesthesia to maintain the femoral head in the proper position without damaging it. Arthrography is a useful tool to evaluate the success of the reduction. The cast is applied to the reduced hip for a period of approximately 12 weeks. If closed reduction cannot be accomplished at this stage, open reduction may be necessary [1]. 


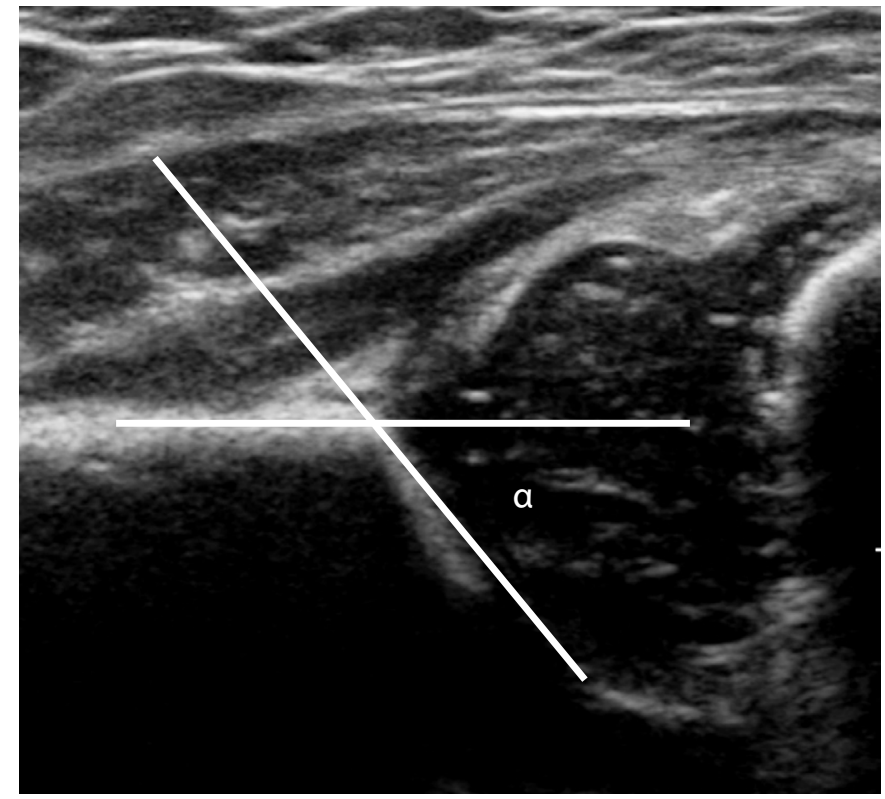

Fig. 6. Physiologically immature hip. Ultrasonography of a 2-month-old girl shows that the a angle is abnormal, measuring $56^{\circ}$.

Open reduction is usually required above the age of 2 years, mostly by femoral osteotomy to relieve pressure over the femoral head and to reshape the acetabulum. The patient is usually immobilized in a spica cast for $6-12$ weeks [1].

\section{Ultrasound for Transient Synovitis of the Hip and Septic Arthritis in Children}

\section{Transient Synovitis}

Transient synovitis of the hip is the most common cause of acute hip pain and limping. It typically occurs in children aged 3-8 years with localized pain in the groin region, anterior thigh, or medial knee [10]. Because the imaging results are similar to those of other causes of acute hip pain in children, the diagnosis is essentially made by exclusion. The main differential diagnoses are septic arthritis [11] and juvenile rheumatoid arthritis [10].

\section{Ultrasound Examination}

Hip US must be performed in the neutral position (hip extended with slight external rotation) using an anterior approach in the parasagittal plane along the long axis of the femoral neck. It may be useful to compare the resulting image with that of the contralateral normal hip (Fig. 8B). On US examination, joint effusion can be seen between the anterior and posterior layers of the anterior joint capsule (Fig. 8A) $[10,12]$. In children, the distance between the

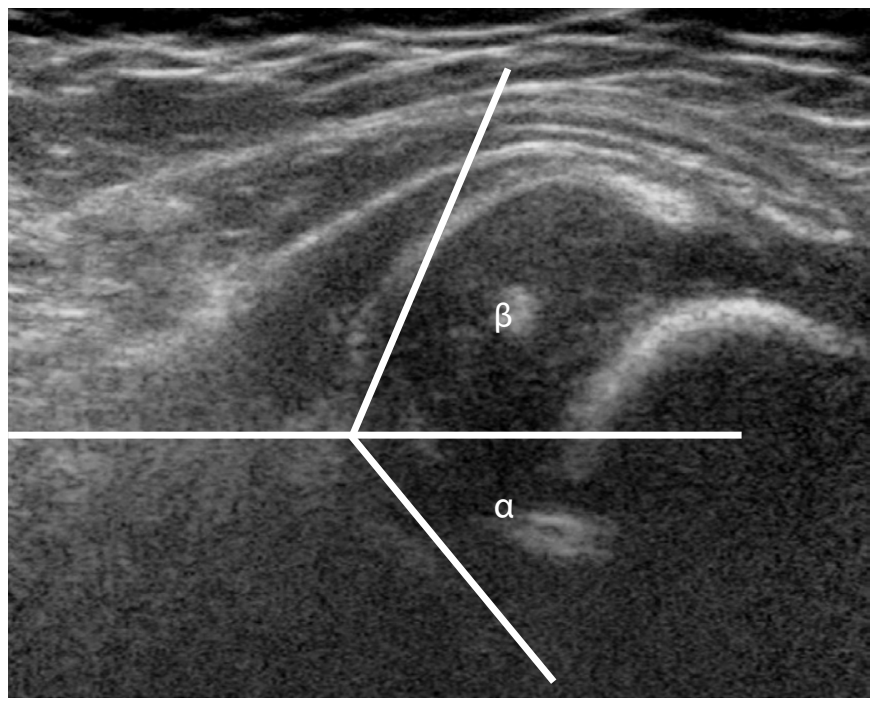

Fig. 7. Developmental dysplasia of the hip. Ultrasonography of a 5-month-old girl shows that the $\alpha$ and $\beta$ angles are abnormal, measuring $46^{\circ}$ and $65^{\circ}$, respectively.

outer margin of the hip capsule and the surface of the femoral neck should not be greater than $5 \mathrm{~mm}$, or more than $2 \mathrm{~mm}$ thicker than the contralateral normal side [13].

\section{Septic Arthritis}

Septic arthritis typically affects the hip, knee, shoulder, elbow, and ankle. Staphylococcus aureus is the most common causative organism. In neonates, group B streptococci and coliform bacteria were previously common causative organisms. In children aged from 3 months to 5 years, Haemophilus influenzae is an important cause, but the incidence has declined considerably due to the use of vaccinations. US of septic arthritis shows hypoechoic joint fluid with or without echogenic debris in patients with clinical signs of joint infection. US can be used for the guided aspiration of joint fluid for the early diagnosis and treatment of septic arthritis $[13,14]$. Additionally, power Doppler may show increased capsular vascularity [15].

\section{Ultrasound of the Neonatal Spine}

\section{Technique and Indications}

Spinal US is performed in both the longitudinal and transverse planes using a linear 5-12 MHz transducer (Fig. 9A, B). The vertebral level is usually determined by counting up from the L5S1 junction. Generally, the infant is examined in the prone position $[7,16]$. 


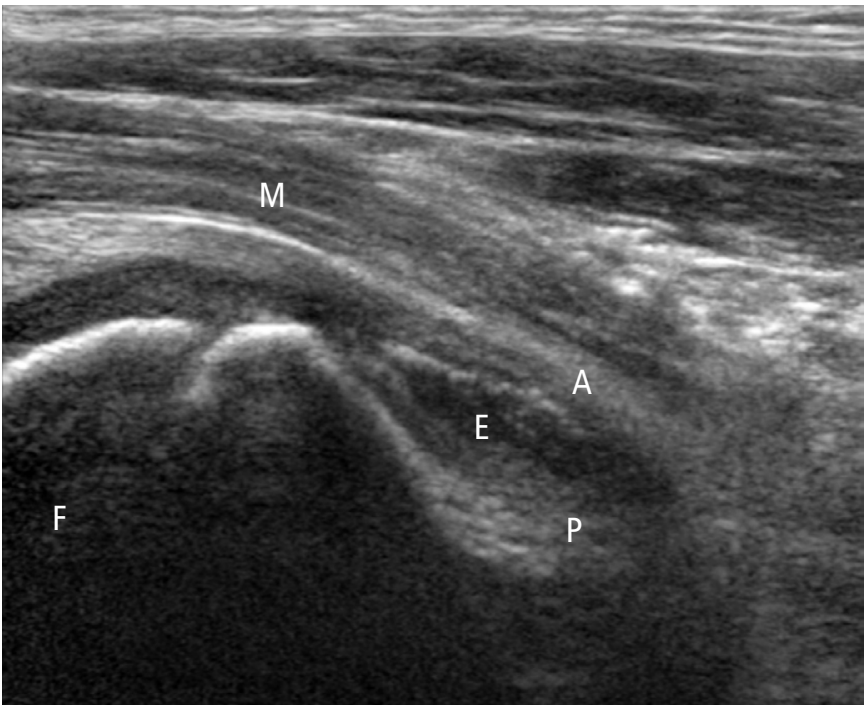

A

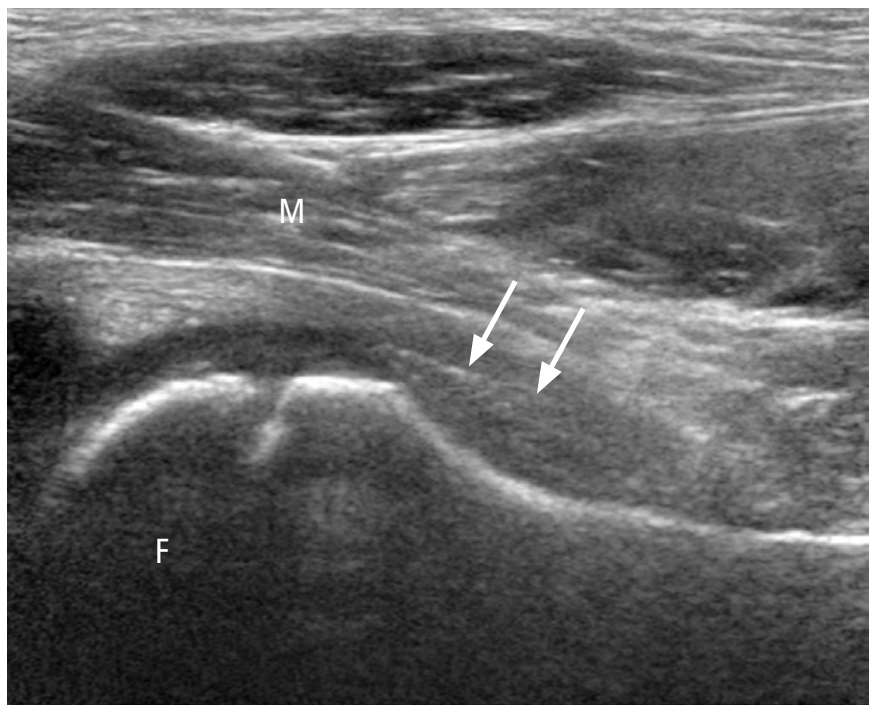

B

Fig. 8. Sagittal ultrasonography of a 6-year-old boy with transient synovitis of the hip and a normal contralateral hip.

A. Sagittal ultrasonography shows that the femoral head (F), iliopsoas muscle (M), anterior (A) and posterior (P) layers of the joint capsules are separated by anechoic effusion (E). B. Sagittal ultrasonography shows the femoral head (F), iliopsoas muscle (M), and both layers of the anterior joint capsule (arrows).

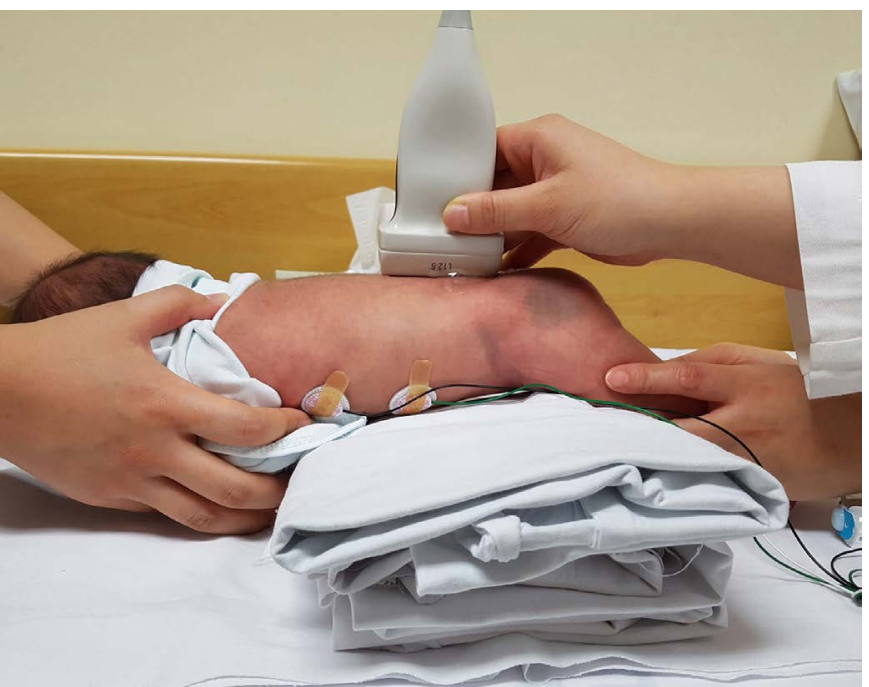

A

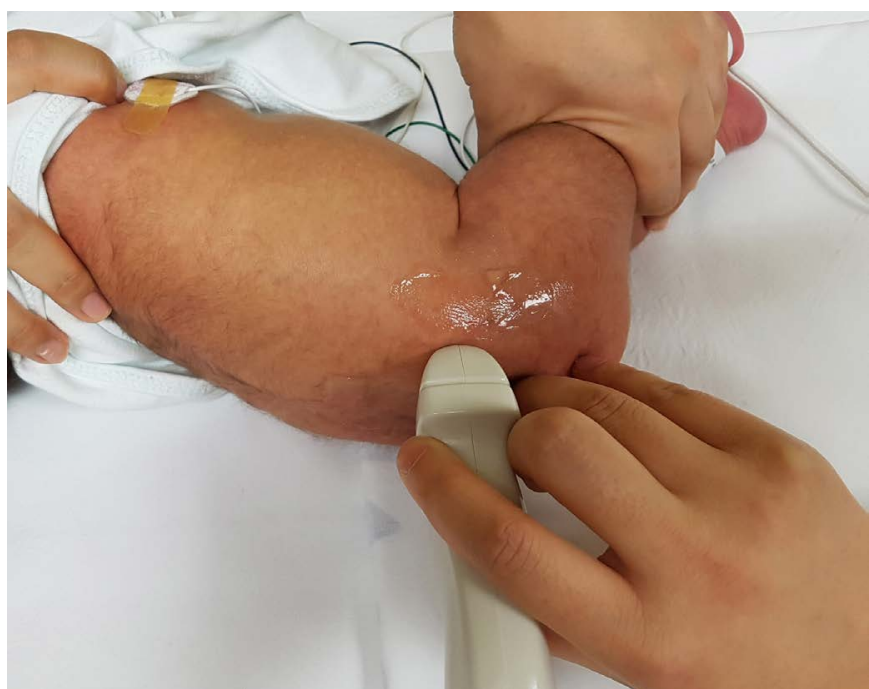

B

Fig. 9. Scanning technique for spinal ultrasonography.

A. Ultrasonography of the spine is performed in the prone position with a small pillow to create kyphosis. The transducer is placed midline over the spinous processes for the longitudinal scan. B. Ultrasonography transducer is placed perpendicular to the midline over the spinous processes for the transverse scan.

There are two main indications for lumbar spinal US in infants. Spinal US is used to evaluate midline cutaneous malformations of the back, such as dimples, as well as hemangiomatous or hairy lesions associated with dysraphic anomalies of the spinal cord. Spinal US is also used to determine the reason for the failure of a lumbar puncture and the location from which cerebrospinal fluid (CSF) may be tapped [17]. Low-risk lesions include simple midline dimples $<5 \mathrm{~mm}$ in diameter, located within $2.5 \mathrm{~cm}$ of the anus, without other cutaneous stigmata. High-risk lesions include atypical dimples $>5 \mathrm{~mm}$ in diameter, a location $>2.5 \mathrm{~cm}$ above the anus, 
hairy patches, hemangiomas, skin tags, and cutis aplasia $[6,7,18]$.

\section{Normal Anatomy}

The longitudinal scan shows a hypoechoic tubular spinal cord with an echogenic central echo complex (Fig. 10A), representing the central canal. On histologic analysis, the central canal corresponds to the border between the myelinated ventral white commissure and the central portion of the anterior median fissure. In healthy newborns, the central canal is not filled with CSF but is instead overgrown with glial fibrils [6].

The caudal end of the spinal cord corresponds to the conus medullaris, which continues into the filum terminale (Fig. 10A). In healthy newborns, the tip of the conus medullaris is no lower than the top of $L 3$ in a term infant (Video clip 1), or the bottom of $L 3$ in a preterm infant [19]. The filum terminale appears as a thin cord-like echogenic structure, forming a parallel line extending from the conus medullaris. The filum terminale is surrounded by the nerve roots of the cauda equina, and it is sometimes difficult to differentiate the filum terminale from the nerve roots [6].

An axial scan of the spinal cord shows a hypoechoic spinal cord with an echogenic central echo complex and paired dorsal and ventral nerve roots within the anechoic subarachnoid space (Fig. 10B) [6].

\section{Normal Variants}

Several common normal variants that may be confused with certain disorders on lumbar spine US are discussed below, including transient dilatation of the central canal, ventriculus terminalis, prominent filum terminale, filar cysts, cauda equina pseudomasses, and pseudosinus tract [6].

\section{Transient dilatation of the central canal}

In newborns, a slight dilatation of the central canal of the spinal cord may be detected (Fig. 11). This is viewed as an incidental

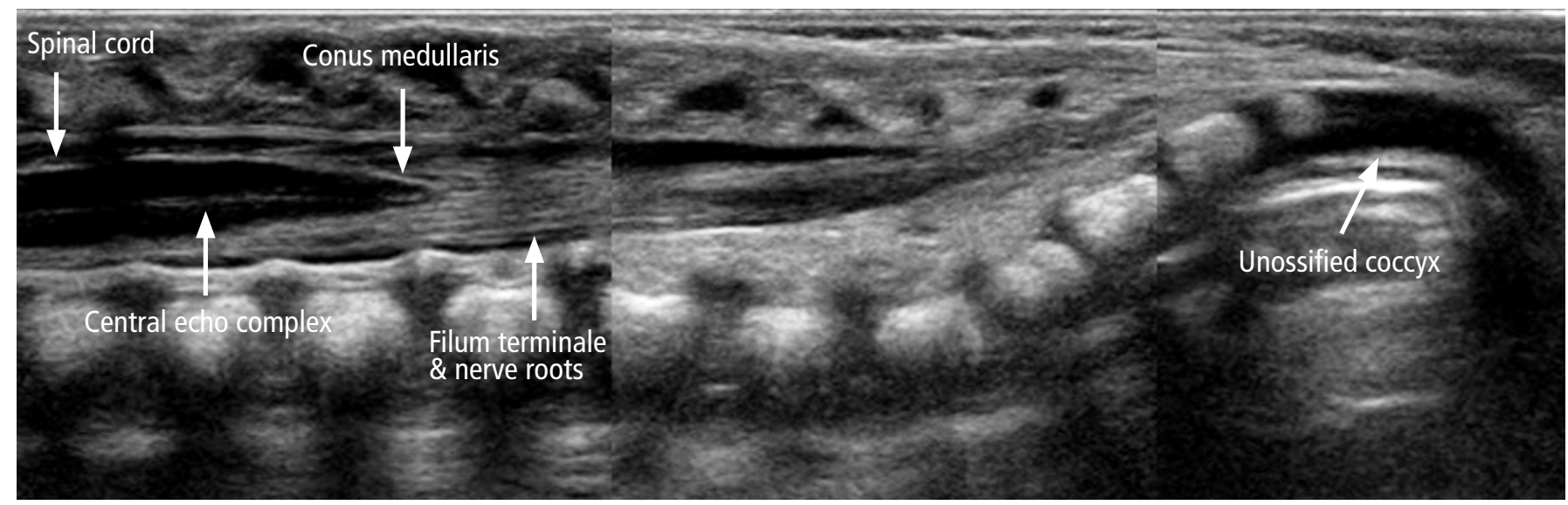

A

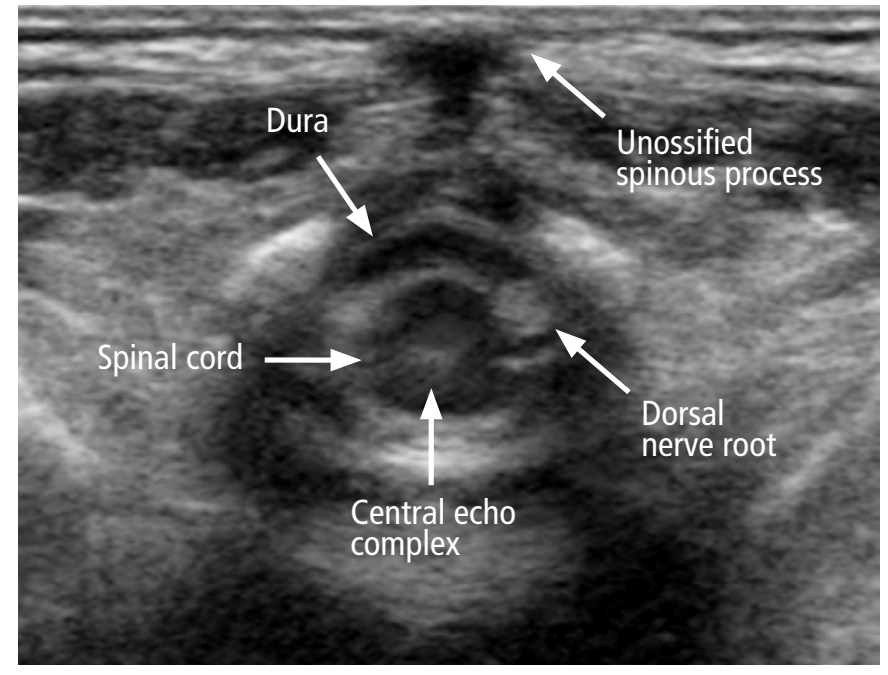

B
Fig. 10. Longitudinal and transverse scan through the spines of a 2-week-old boy.

A. Longitudinal lumbar ultrasonography shows normal anatomy, as labeled. B. Transverse lumbar ultrasonography shows normal anatomy, as labeled. 
finding in healthy newborns and almost disappears during the first weeks of postnatal life [6].

\section{Ventriculus terminalis}

The ventriculus terminalis is a small, ependyma-lined, oval, cystic structure positioned at the distal cord (Fig. 12). This structure extends over 8-10 $\mathrm{mm}$ and has a transverse diameter of 2-4 mm. The ventriculus terminalis develops during embryogenesis due to incomplete fetal regression of the embryonic terminal ventricle

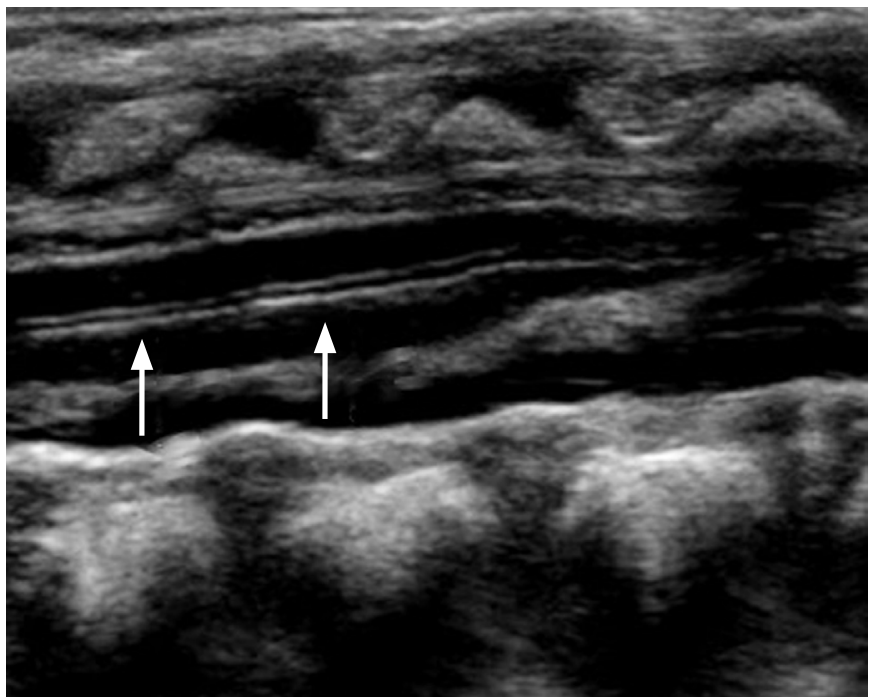

Fig. 11. Transient dilatation of the central canal. Longitudinal scan shows transient dilatation of the central canal (arrows) in a 4-dayold boy with a simple sacral dimple and normal motor development.

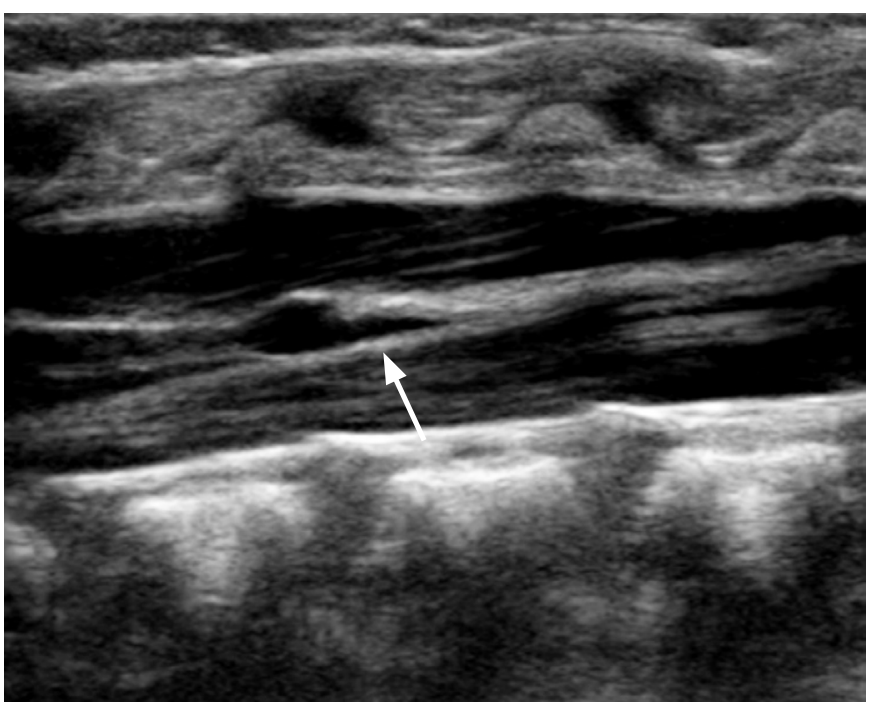

Fig. 12. Ventriculus medullaris. Longitudinal ultrasonography in a 3-week-old girl shows a well-defined, fusiform-shaped cystic lesion (arrow) in the distal portion of the conus medullaris. during the first weeks after birth in the conus medullaris. This variant causes no clinical symptoms [6].

\section{Prominent filum terminale}

If the filum terminale is observed to be particularly echogenic in comparison with other nerve roots (Fig. 13), this may be a cause of concern. It can be distinguished as normal variants by a typical midline course and a thickness of less than $2 \mathrm{~mm}$ [7].

\section{Filar cysts}

A filar cyst is located in the filum terminale and has a fusiform shape and the well-defined, hypoechoic appearance of a simple cyst (Fig. 14). A filar cyst is distinguished from the ventriculus medullaris by its location just below the conus medullaris. It is a normal variant and has no known clinical significance. Therefore, a filar cyst does not require additional imaging [7].

\section{Pseudomass due to positional nerve root clumping}

When a newborn is scanned in the decubitus position, positional clumping of the nerve roots can occur (Fig. 15). However, such masses disappear when the child changes to the prone position [7].

\section{Pseudosinus tract}

The pseudosinus tract is seen on US as a residual cord-like region composed of fibrous tissue extending from a skin dimple to the coccyx (Fig. 16). True dermal sinus tracts do not occur at the tip of the coccyx but are typically found in a more cranial location. However, careful evaluation should be performed for any fluid or

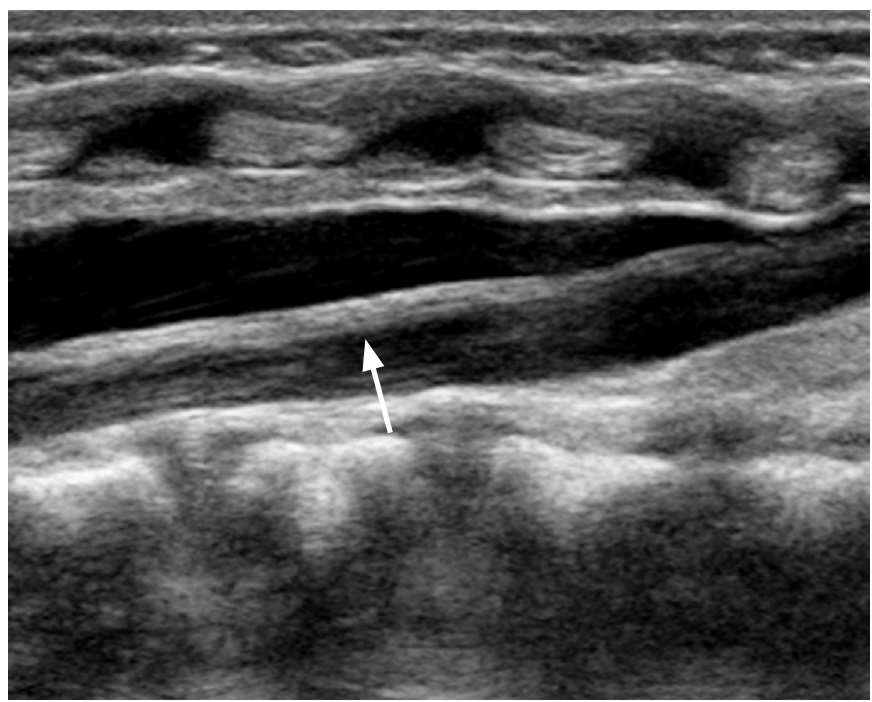

Fig. 13. Prominent filum terminale. Longitudinal ultrasonography in a 4-week-old girl shows prominent filum terminale $(<1 \mathrm{~mm})$ (arrow) at L5-S1. 


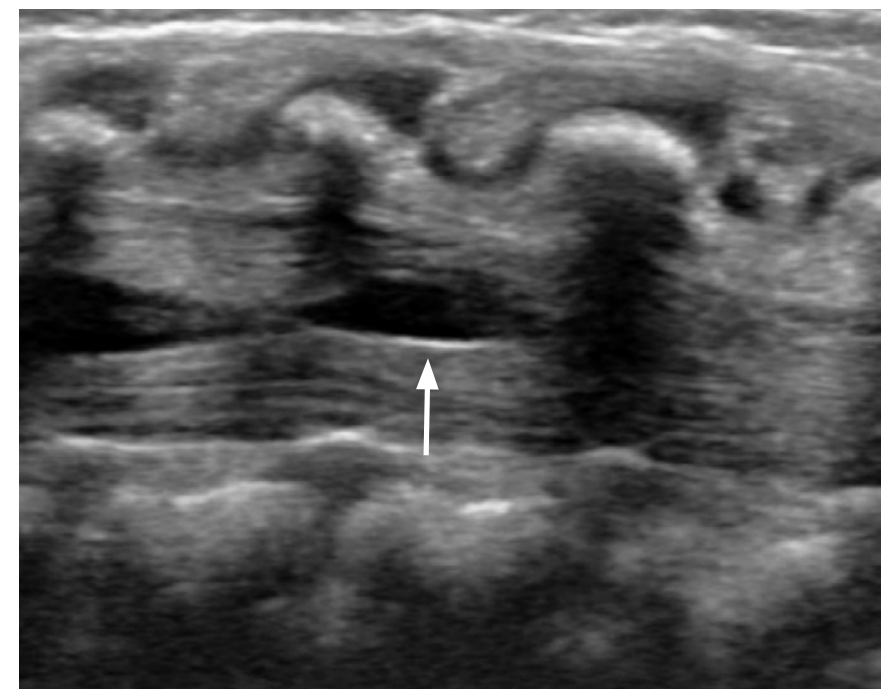

A

Fig. 14. Filar cyst in a 2-week-old girl.

A. Longitudinal ultrasonography shows a well-defined, fusiform-shaped cystic lesion (arrow) within the filum, just below the conus medullaris. B. Transverse ultrasonography shows a cystic lesion (arrow).

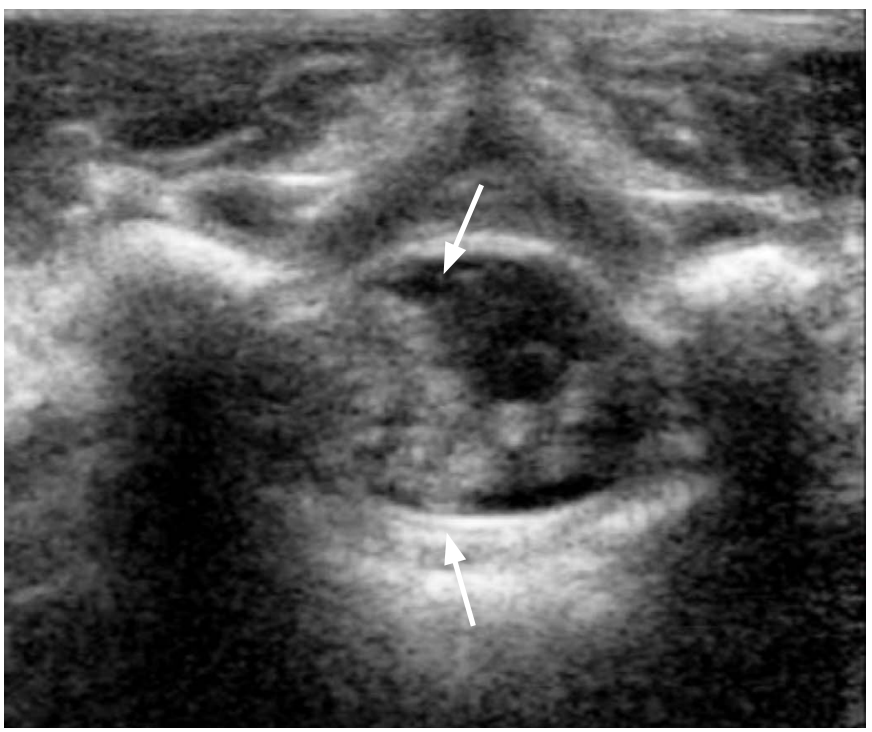

A

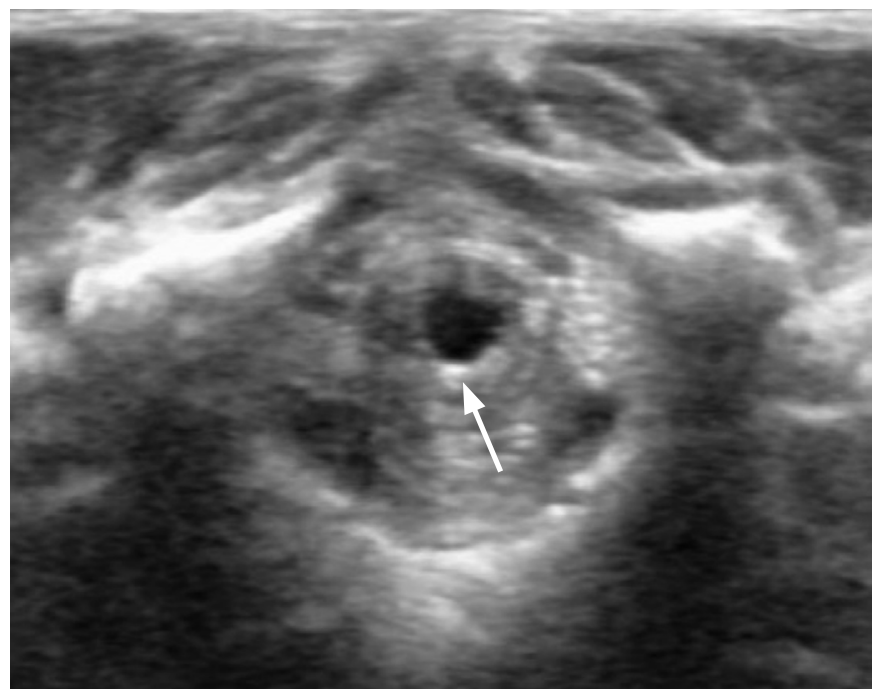

B 
tissue masses. Myelomeningocele and myelocele show a mass located on the back but are without a skin covering $[6,20]$.

\section{Tethered cord}

Tethered cord is caused by the incomplete involution of the terminal spinal cord. Using real-time US, a low-lying conus below the L2L3 disk space with an absence of normal nerve root motion can be diagnosed as a tethered cord (Fig. 17B). Traction on the abnormally anchored filum terminale and adjacent nerve roots can initiate

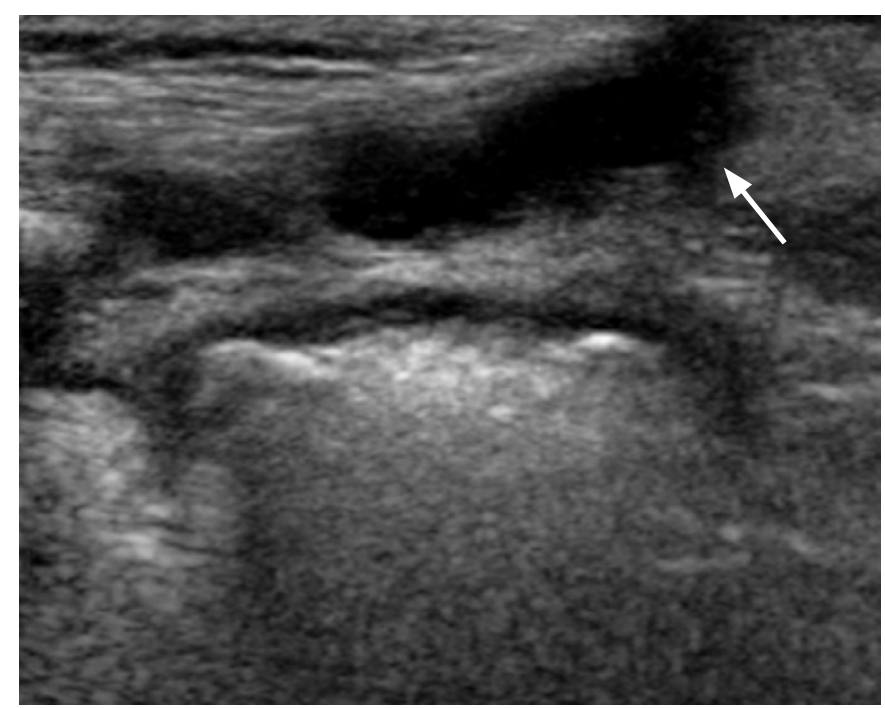

Fig. 16. Pseudosinus tract in a 4-week-old infant with a sacral dimple. Sagittal ultrasonography of the coccygeal region shows a hypoechoic line extending from the skin dimple to the distal coccyx (arrow). This finding is considered a normal variant and is not associated with spinal dysraphic anomalies.

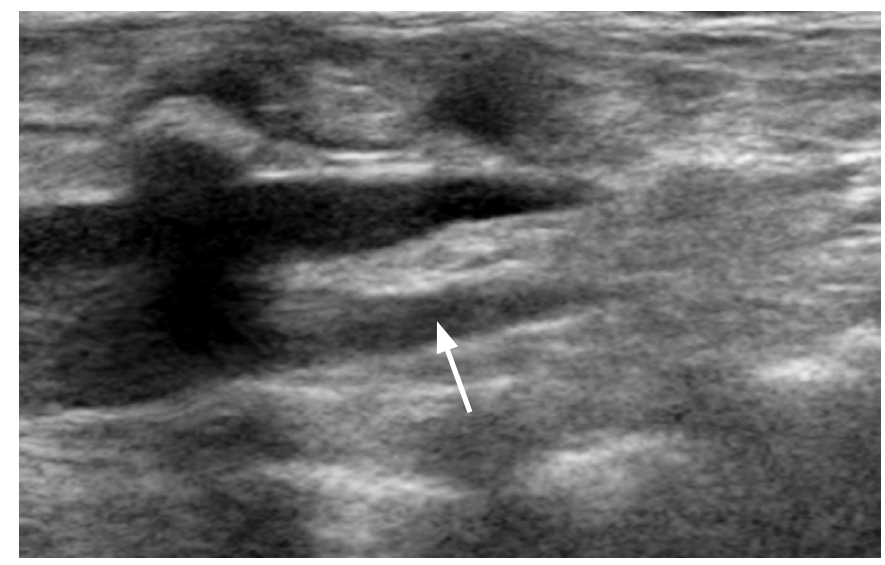

A

Fig. 17. Tethered cord in a 5-week-old girl.

A. Longitudinal ultrasonography shows a hyperechoic thickened filum terminale (arrow) at the L5-S1 level. B. Longitudinal ultrasonography shows a low-lying conus (arrow) at the L4 vertebra and a thickened, echogenic fatty filum.

e-ultrasonography.org

Ultrasonography 36(3), July 2017

\section{Myelocele and myelomeningocele}

During embryogenesis, the dorsally localized failure of fusion of the neural folds leads to myelocele and myelomeningocele. Severe neurologic disturbances, mainly of the lower extremities (such as paresis or paralysis and bladder or bowel dysfunction), can occur in patients with myelocele and myelomeningocele, as well as secondary hydrocephalus after repair [6].

The purpose of spinal US in patients with myelocele or myelomeningocele is the recognition of associated malformations.

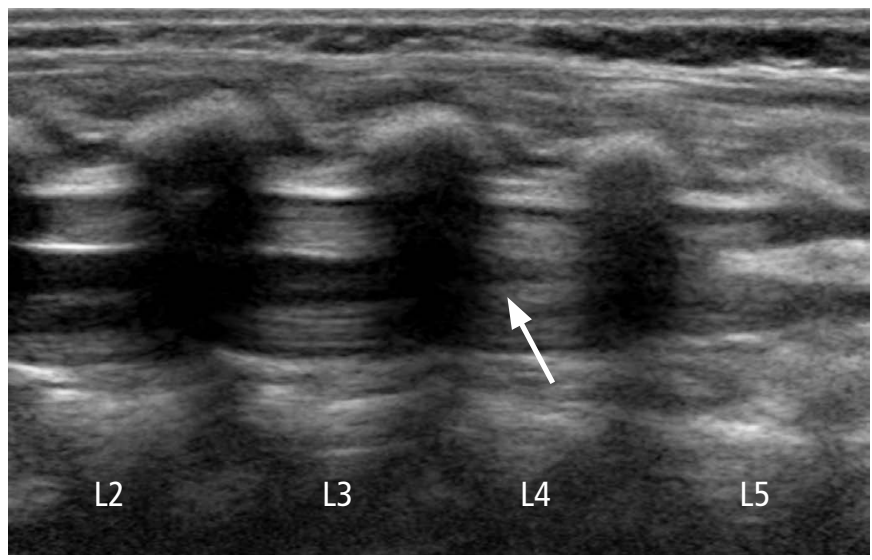

B

clinical symptoms at any age, such as difficulties in ambulation, weakness, abnormal reflexes, stiffness, bladder dysfunction, and, less

Other associated spinal findings include a thickened filum terminale, spinal dysraphism, congenital spinal lipomas or dermoids, syringomyelia, scoliosis, myelocele, and dermal sinus tracts. Other non-neurologic anomalies, including tracheoesophageal fistula, VATER syndrome, and congenital heart disease, are also commonly

\section{Spinal lipoma}

Spinal lipomas are composed of normal fat that may change in size with increased weight and tend to grow significantly during the first year of life. Spinal lipomas appear on ultrasound as an echogenic intraspinal mass adjacent to the deformed spinal cord. They may be intradural, extradural, or a combination thereof. Eighty-four percent of lipomas contain not only fat but also neural tissue or meninges. Associated features include tethered cord, dysraphism (4\%), fatty filum or lipoma of the filum (12\%), and vertebral anomalies. Treatment consists of resection, when possible, or debulking $[6,20]$. 
These malformations consist of tethering of the spinal cord, diastematomyelia, hydromyelia or syringomyelia, Chiari II syndrome, and arachnoid cysts [6].

\section{Dorsal dermal sinus}

A dorsal dermal sinus is observed as an epithelium-lined tract between the skin and the spinal cord, cauda equina, or arachnoid. Dorsal dermal sinus is caused by incomplete separation of the superficial ectoderm from the neural ectoderm at a circumscribed point. A dorsal dermal sinus may appear as a small dimple or pinpoint ostium with or without an area of hyperpigmentation, hair, or angiomatous skin, usually in a midline or, rarely, in a paramedian location. Various infections, including recurrent meningitis, epidural or subdural abscess, as well as abscess formation at the conus medulla of the spinal cord, can occur via a dorsal dermal sinus [6].

\section{Conclusion}

The clinical examination of the hips in all infants, immediately after birth and at each scheduled well child visit up to the age of 1 year, is recommended in current published guidelines. Children who have intermediate findings or increased risk factors with a normal examination should undergo US imaging at the age of 3-6 weeks depending on the risk factors present, or plain radiographs at 4-5 months of age if reliable US is unavailable. The goals of treatment are to normalize the alignment between the femoral head and acetabulum, and the distribution of biomechanical forces about the hip joint. The ultimate aim of treatment is to reduce the incidence of symptoms and secondary degeneration later in life.

Transient synovitis of the hip is one of the most common causes of hip pain and limping in young children. It has an acute onset without trauma and is self-limiting after conservative therapy. Ultrasound is the best imaging method for the diagnosis of transient synovitis because it involves a brief examination and the patient is not exposed to ionizing radiation. The US examination has the goal of identifying effusion in the anterior recess of the joint capsule. Comparing the image with that of the contralateral normal hip may be useful.

Neonatal spinal US is a useful screening technique for congenital spinal anomalies and can also characterize normal anatomical structures and normal variants that may resemble disorders. Familiarity with these findings can help to prevent misinterpretation and inappropriate referrals. In patients with normal findings, no further imaging examinations are necessary. In patients with spinal malformations on US, a further examination can be performed at the time of the elective surgical intervention. In addition, in complex spinal malformations, the role of US is to allow the detection of associated anomalies.

ORCID: Yeo Ryang Kang: http://orcid.org/0000-0003-1161-8415; Joonbum Koo: http://orcid.org/0000-0002-1884-7758

\section{Conflict of Interest}

No potential conflict of interest relevant to this article was reported.

\section{Supplementary Material}

Video clip 1. Longitudinal lumbar sonogram of a 3-week-old boy with a linear 5-12 MHz probe shows normal anatomy (https://doi. org/10.14366/usg.16051.v001).

\section{References}

1. Alsaleem M, Set KK, Saadeh L. Developmental dysplasia of hip: a review. Clin Pediatr (Phila) 2015;54:921-928.

2. Starr V, Ha BY. Imaging update on developmental dysplasia of the hip with the role of MRI. AJR Am J Roentgenol 2014;203:13241335.

3. Paton RW, Choudry Q. Neonatal foot deformities and their relationship to developmental dysplasia of the hip: an 11-year prospective, longitudinal observational study. J Bone Joint Surg Br 2009;91:655-658.

4. Matrawy KA, Nouh MR. Ultrasound screening for developmental dysplasia of the hip and its socioeconomic impact: experience of tertiary care health level. Alexandria J Med 2014;50:25-29.

5. Omeroglu $\mathrm{H}$. Use of ultrasonography in developmental dysplasia of the hip. J Child Orthop 2014;8:105-113.

6. Unsinn KM, Geley T, Freund MC, Gassner I. US of the spinal cord in newborns: spectrum of normal findings, variants, congenital anomalies, and acquired diseases. Radiographics 2000;20:923938.

7. Lowe LH, Johanek AJ, Moore CW. Sonography of the neonatal spine: part 1, Normal anatomy, imaging pitfalls, and variations that may simulate disorders. AJR Am J Roentgenol 2007;188:733-738.

8. Graf R. Hip sonography: diagnosis and management of infant hip dysplasia. 2nd ed. Berlin: Springer, 2006.

9. American Institute of Ultrasound in Medicine. AIUM practice guideline for the performance of an ultrasound examination for detection and assessment of developmental dysplasia of the hip. J Ultrasound Med 2013;32:1307-1317.

10. Pauroso S, Di Martino A, Tarantino CC, Capone F. Transient synovitis of the hip: Ultrasound appearance: mini-pictorial essay. J Ultrasound 2011;14:92-94.

11. Kwack KS, Cho JH, Lee JH, Oh KK, Kim SY. Septic arthritis versus transient synovitis of the hip: gadolinium-enhanced MRI finding of decreased perfusion at the femoral epiphysis. AJR Am J Roentgenol 
2007; 189:437-445.

12. Wingstrand H, Egund N, Carlin NO, Forsberg L, Gustafson T, Sunden G. Intracapsular pressure in transient synovitis of the hip. Acta Orthop Scand 1985;56:204-210.

13. Chau CL, Griffith JF. Musculoskeletal infections: ultrasound appearances. Clin Radiol 2005;60:149-159.

14. Gutierrez K. Bone and joint infections in children. Pediatr Clin North Am 2005;52:779-794.

15. Strouse PJ, DiPietro MA, Teo EL, Doi K, Chrisp CE. Power Doppler evaluation of joint effusions: investigation in a rabbit model. Pediatr Radiol 1999;29:617-623.

16. Nair N, Sreenivas M, Gupta AK, Kandasamy D, Jana M. Neonatal and infantile spinal sonography: a useful investigation often underutilized. Indian J Radiol Imaging 2016;26:493-501.

17. Coley BD, Shiels WE 2nd, Hogan MJ. Diagnostic and interventional ultrasonography in neonatal and infant lumbar puncture. Pediatr Radiol 2001;31:399-402.

18. Kriss VM, Desai NS. Occult spinal dysraphism in neonates: assessment of high-risk cutaneous stigmata on sonography. AJR Am J Roentgenol 1998;171:1687-1692.

19. Hill CA, Gibson PJ. Ultrasound determination of the normal location of the conus medullaris in neonates. AJNR Am J Neuroradiol 1995; 16:469-472.

20. Lowe LH, Johanek AJ, Moore CW. Sonography of the neonatal spine: part 2, Spinal disorders. AJR Am J Roentgenol 2007;188:739744. 